\title{
ORIGINAL ARTICLE Use of medicines, adherence and attitudes to medicines among persons with chronic spinal cord injury
}

\author{
H Høgholen ${ }^{1,2}$, A Storhaug ${ }^{2}, \mathrm{~K}_{\text {Kvernrød }}^{2}$, E Kostovski ${ }^{3,4}$, KK Viktil ${ }^{1,5}$ and L Mathiesen ${ }^{2}$
}

Study design: A cross-sectional study.

Objectives: To describe the use of medicines and adherence among persons with spinal cord injury (SCI). Further, to examine the influence of pain, spasms and beliefs about medicines on adherence.

Setting: Sunnaas Rehabilitation Hospital, Norway.

Methods: Persons ( $\geqslant 18$ years) with chronic SCl (more than 1-year post injury), using at least one drug regularly, and admitted for a follow-up stay at Sunnaas Rehabilitation Hospital were included. Participants were interviewed about their drug regimen and filled out validated self-report questionnaires: Morisky Medication Adherence Scale (MMAS-8), beliefs about medicines questionnaire (BMQ), visual analogue scale (VAS) for pain and modified Penn spasm frequency scale (mPSFS).

Results: The 105 participants used in average 4.2 drugs regularly (range, 1-15), and 70\% reported high or moderate adherence to their treatment. Of the 39 participants using oral spasmolytics, 74\% reported high or moderate adherence to these drugs. A total of $97 \%$ of the participants reported high perceptions of necessity to their treatment and $54 \%$ reported a high level of concern.

Conclusion: The persons with $\mathrm{SCl}$ included in this study used in average the same number of regular drugs compared to persons with other chronic conditions. Regardless of high overall adherence, the participants were more concerned about their medicines compared to other patient groups. Further studies are required for understanding adherence and attitudes toward medicines in this population, especially to help the persons with chronic SCI feel safe about their drug regimen.

Spinal Cord (2018) 56, 35-40; doi:10.1038/sc.2017.95; published online 29 August 2017

\section{INTRODUCTION}

A spinal cord injury (SCI) can, in addition to loss of sensibility and motor function, affect most body systems, causing neuropathic pain, spasticity and cardiovascular, bowel and bladder complications, all of which can result in a complex medication regimen. ${ }^{1-3}$ This often implies a challenge with regard to adherence. ${ }^{4}$

Adherence is described by the World Health Organization (WHO) as 'the extent to which a person's behavior-taking medication, following a diet, and/or executing lifestyle changes, corresponds with agreed recommendations from a healthcare provider'. ${ }^{4}$ There are several factors that can have an impact on adherence. A person can intentionally be non-adherent because of personal beliefs or experience. One can also be unintentionally non-adherent by forgetting to take their medicine or because of language issues. ${ }^{5}$ It is known that $\sim 50 \%$ of persons with chronic conditions are non-adherent to their long-term therapies. ${ }^{4}$ Non-adherence can have a negative impact on the health benefit of a medication regimen and contribute to increased morbidity and mortality, and increased healthcare costs. ${ }^{4}$

There is limited knowledge about the extent of use of medicines and also the adherence and attitudes to medicines among persons with chronic SCI ( $>1$-year post injury). ${ }^{6}$ To our knowledge there is only one previous study examining adherence to oral spasmolytic drugs among persons with SCI. ${ }^{7}$
A better understanding of these challenges could help to customize and adapt counseling and level of assistance with medications to the person's needs. The main objectives of this study were to describe the medication use in the population of persons with chronic SCI, and to examine adherence to regular medicines, and to oral spasmolytic drugs in particular. Our secondary objective was to examine if pain, spasms and beliefs about medicines are associated with adherence.

\section{MATERIALS AND METHODS}

\section{Participants}

The study was performed at the Department for follow-up at Sunnaas Rehabilitation Hospital, Nesoddtangen, Norway. There are $\sim 1500-2500$ persons living with SCI in Norway and Sunnaas Rehabilitation Hospital is one of three hospitals in Norway offering rehabilitation to these persons. ${ }^{8}$ Sunnaas is also the country's largest specialized hospital in physical medicine and rehabilitation with a catchment area of about 2.9 million people (55\% of the Norwegian population). In addition to its regional function, Sunnaas also has a national lifetime healthcare responsibility for all persons with SCI in Norway. Follow-up stays are voluntary, and are usually from 2 days up to several weeks of duration.

Participants over 18 years, with chronic SCI, that is, at least 1 -year post injury, using at least one drug regularly and being admitted for a follow-up stay between August 2015 and January 2016 were included. Participants already included were not included again if they were readmitted in the study period. Participants were excluded if they did not have adequate knowledge of

\footnotetext{
${ }^{1}$ School of Pharmacy, University of Oslo, Oslo, Norway; ${ }^{2}$ Hospital Pharmacies Enterprise, South Eastern Norway, Oslo, Norway; ${ }^{3}$ Science Department, Sunnaas Rehabilitation Hospital, Nesoddtangen, Norway; ${ }^{4}$ Faculty of Medicine, Department of Physical Medicine and Rehabilitation, University of Oslo, Oslo, Norway and ${ }^{5}$ Diakonhjemmet Hospital Pharmacy, Oslo, Norway

Correspondence: H Høgholen, Hospital Pharmacies Enterprise, South Eastern Norway, Sykehusapotekene Oslo, Kirkeveien 166, 0450 Oslo, Norway.

E-mail: anna.helene.hoghole@sykehusapotekene.no

Received 4 May 2017; revised 10 July 2017; accepted 11 July 2017; published online 29 August 2017
} 
Norwegian language to be able to complete the questionnaires used in the study.

\section{Measures}

A modified form based on the validated method for integrated medicines management (IMM) was used for collecting information about the participants' medication regimen. ${ }^{9}$ The modified form was tested in a pilot study with five participants, but not formally validated. In the first part of the form, participants were asked to provide information about which medicines they used regularly and which they used on demand and at which dosage. The medications were registered according to the anatomical therapeutic chemical (ATC) classification. ${ }^{10}$ Furthermore, they reported the use of spasmolytic drugs (either baclofen or tizanidine), the duration of treatment for all their medicines and if they needed assistance in taking them, and whether the medicines were prescribed by a general practitioner (GP) or a specialist. The second part of the form focused on follow-up questions regarding adherence and had open-end questions about the participants' experience with their medicine use.

Adherence was measured with the Morisky Medication Adherence Scale (MMAS-8). ${ }^{1-13}$ The validated Norwegian translation of MMAS-8 and permissions to use the scale in this study was provided by Prof. Donald E Morisky. The scale contains eight questions assessing medication taking behavior and other aspects around adherence. Two MMAS- 8 scales were used in this study, one to measure adherence to all the participants regularly used drugs and one to measure adherence to spasmolytic drugs, that is, baclofen and tizanidine.

Beliefs about medicines questionnaire (BMQ $)^{14}$ consists of two sections, BMQ-Specific, which assess beliefs about necessity and concern for personal medicines, and BMQ-General, which assesses general beliefs about overuse and harm of medicines. All the questions in the BMQ-Specific and the BMQGeneral are of the five-point Likert-scale. ${ }^{14}$ The total score was divided with the amount of statements in the subscales to get a mean score. Higher scores represented higher level of agreement in the dimensions measured by the different subscales. In addition, the scores from the necessity and concern subscale were presented by dichotomizing the participants into high/low necessity and high/low concern based on whether they scored over or below the midpoint for the BMQ-Necessity and BMQ-Concern subscale. Based on these dichotomized scores, the participants were then categorized into four different groups: accepting (high necessity and low concern), ambivalent (high necessity and high concern), indifferent (low necessity and low concern) and sceptical (low necessity and high concern). ${ }^{14}$

In addition, the participants were asked to provide information about the degree of pain and the spasticity they were experiencing, to see if these two variables could be associated with adherence. Pain was measured with visual analogue scale (VAS). ${ }^{15}$ The score was announced in millimeters $(0-100 \mathrm{~mm})$ and was divided in three different levels of pain according to an article by Collins et al. ${ }^{16}$ published in Pain (1997), and predetermined in the protocol: mild pain (score under $30 \mathrm{~mm}$ ), moderate pain (score between $30 \mathrm{~mm}$ and $54 \mathrm{~mm}$ ) and severe pain (score over $54 \mathrm{~mm}$ ). Participants in this study were asked to fill out two separate VAS, one for the level of pain they were experiencing at the point of the interview and another one to measure pain level 14 days before the interview.

Frequency and severity of spasms were measured by modified Penn spasm frequency scale (mPSFS) ${ }^{17}$ mPSFS consists of two scales, where the first one is a five-point scale for assessing the frequency of spasticity and the second scale a three-point scale for assessing the severity of spasticity. ${ }^{17}$

\section{Procedure}

Potential participants were identified by the study pharmacist or a nurse. All participants provided written, informed consent before being included in the study.

The following data were provided from the medical records for all participants before the interview: age, gender, cause and type of SCI (neurological level, traumatic or atraumatic, tetraplegia or paraplegia, complete or incomplete), American Spinal Injury Association Impairment Scale (AIS) grade, ${ }^{18}$ time since injury (years), relevant medical history and time of their last admission to Sunnaas Rehabilitation Hospital.
The study pharmacist interviewed the participants using the first part of the modified IMM-form described above. Then the participants filled in self-report questionnaires, as described. These were filled out by the participant in their room without the study pharmacist being present. Participants unable to fill out the questionnaires by themselves because of paralysis, received assistance from either a personal assistant or the study pharmacist. Lastly, the study pharmacist completed the interview using the second part of the modified IMM-form and the participants also got a chance to ask questions or make comments.

The time for the interview was scheduled by the study pharmacist together with the participant, and the interview took place in the participant's hospital room.

\section{Statistical analysis}

Data were analyzed using SPSS 22.0 for Windows. Association between adherence and relevant variables were tested using $\chi^{2}$ tests. The variables tested

Table 1 Characteristics for the 105 participants with $\mathrm{SCl}$ included in the study

\begin{tabular}{lrrrr} 
& \multicolumn{4}{c}{$N=105$ participants } \\
\cline { 2 - 5 } & $\mathrm{N}$ & $\%$ & Mean (s.d.) & Median (range) \\
& & & & \\
Gender & 66 & 62.8 & & \\
$\quad$ Male & 39 & 37.1 & & \\
Female & & & & \\
& & & $53(15.4)$ & \\
Age & 11 & 10.4 & & \\
$16-30$ & 22 & 10.9 & & \\
$31-45$ & 34 & 32.4 & & \\
$46-60$ & 31 & 29.5 & & \\
$61-75$ & 7 & 6.7 &
\end{tabular}

Years since injury

$19(14)$

$15(2-54)$

AIS

A

B

C

D

E

Unkown

6.7

9.5

25.7

0

Neurological category

C1-C4 A,B,C

C5-C8 A,B,C

T1-S3 A,B,C

All AIS D

Unknown

35.2

Type of injury

Incomplete

Complete

Unknown

52

49.5

36.2

14.3

\section{Level of injury}

Paraplegic

Tetraplegic

Uknown

57

54.3

$40 \quad 38.1$

Medicines

Regular

On demand

$4.2(2.8)$

$4(1-15)$

Abbreviation: AIS,

1 (1.3)

$0(0-5)$

Abbreviation: AIS, American Spinal Injury Association Impairment Scale. 
were age, gender, neurological level of injury, AIS grade, time since injury (years), BMQ (each subscale separately), number of regularly used drugs and the level of spasticity (only for those using oral spasmolytic drugs). Variables as age, time since injury, number of regularly used drugs and neurological level of injury were divided into following groups to get a more uniform distribution before the analysis:

Age: $21-46,48-64,65-87$

Time since injury: 1-12, 13-25, 26-55

Number of regularly used drugs: $1-2,3-5,6-15$

Neurological level of injury: C1-C8, T1-T12, L1-S5

A retrospective sensitivity analysis was also performed, where the variables mentioned above were grouped following the standardization as described by Biering-Sorensen et al. ${ }^{18}$

\section{Statement of ethics}

We received ethical approval for this study from the Norwegian Regional Committee for Medical and Health Research Ethics (REC South East, case number 2015/724). We certify that all applicable institutional and governmental regulations concerning the ethical use of human volunteers were followed during the course of this research.

\section{RESULTS}

\section{Participant characteristics}

From a total of 173 persons with SCI admitted for a follow-up stay during the study period, 125 persons met the inclusion criteria. Of these, 112 persons gave their consent to participate. Further, two participants were excluded because they did not show up for the interview, two withdrew their consent to participate and three participants were excluded after inclusion because they did not continue to meet the inclusion criteria at the point of interview.

Table 2 Overview of the 10 most used regular and on demand APIs expressed as number of prescriptions per API and percentage of participants using the drug

\begin{tabular}{|c|c|c|}
\hline$A P I$ & Prescriptions, $\mathrm{n}$ & $\%^{a}$ \\
\hline \multicolumn{3}{|l|}{ Used regularly } \\
\hline Baclofen (ATC group M) & 35 & 33.3 \\
\hline Methenamine (ATC group J) & 30 & 28.6 \\
\hline Pregabalin (ATC group N) & 23 & 21.9 \\
\hline Gabapentin (ATC group N) & 22 & 20.9 \\
\hline Amitriptyline (ATC group N) & 19 & 18.1 \\
\hline Solifenacin (ATC group G) & 16 & 15.4 \\
\hline Acetylsalisylic acid (ATC group B) & 15 & 14.3 \\
\hline Paracetamol (ATC group N) & 13 & 12.4 \\
\hline Furosemide (ATC group C) & 10 & 9.5 \\
\hline Zopiclone (ATC group N) & 10 & 9.5 \\
\hline \multicolumn{3}{|l|}{ Used on demand } \\
\hline Paracetamol (ATC group N) & 14 & 13.3 \\
\hline Tramadol (ATC group N) & 9 & 8.6 \\
\hline Diazepam (ATC group N) & 7 & 6.7 \\
\hline Codeine+Paracetamol (ATC group N) & 7 & 6.7 \\
\hline Zopiclone (ATC group N) & 6 & 5.7 \\
\hline Ibuprofen (ATC group M) & 4 & 3.8 \\
\hline Oxazepam (ATC group N) & 4 & 3.8 \\
\hline Oxycodone (ATC group N) & 4 & 3.8 \\
\hline Diclofenac (ATC group M) & 3 & 2.9 \\
\hline Ketobemidone (ATC group N) & 2 & 1.9 \\
\hline
\end{tabular}

Abbreviations: API, active pharmaceutical ingredient; ATC, anatomical therapeutic chemical classification.

apercent of participants using drug.
Finally, 105 participants completed the study. The participants had a mean age of 53 years and most were males (63\%), Table 1. A total of 24 participants $(23 \%)$ needed assistance in completing the questionnaires, two of them were assisted by a personal assistant and the rest by the study pharmacist. There were no missing answers on any of the questionnaires.

\section{Use of medicines}

The participants used in average 4.2 drugs regularly (range, 1-15; s.d. 2.8 , median 4 ) and one drug on demand (range, $0-5$; s.d. 1.3, median 0 ). We registered a total of 440 prescriptions of regular medicines, corresponding to 129 active pharmaceutical ingredients (API). The most frequent APIs used were drugs for the nervous system (ATC group N), which was used by $30 \%$ of the participants. An overview of the 10 most regularly used APIs is listed in Table 2.

A total of $66 \%$ of the participants used one or more oral drug for pain, including not only drugs classified as analgesics (ATC-group N02), but also pregabalin, gabapentin and amitriptyline, which are APIs often used in treatment of neuropathic pain. At the time of the interview $94 \%$ of the participants reported at least some level of pain, of which $53 \%$ reported intense or moderate pain according to the VAS measurements.

A total of 39 participants (37\%) used oral spasmolytic drugs: 35 of them used baclofen, 7 used tizanidine and 3 participants used a combination of both. A total of $77 \%$ of the participants reported at least some level of spasticity, $18 \%$ of these reported severe spasticity.

Of drugs used on demand, 100 prescriptions were registered, corresponding to 46 various APIs, of which $63 \%$ were drugs for the nervous system (ATC-group N). An overview of the 10 most frequently used APIs on demand is listed in Table 2.

As many as 95 participants (90\%) reported that they did not need assistance to take their medicines. A total of $30 \%(n=31)$ of the participants felt they lacked knowledge about their medicines, and most of them reported that they chose to trust their doctor instead of trying to gain more knowledge.

\section{Adherence}

Results from the MMAS- 8 for all regularly used drugs and oral spasmolytic drugs are presented in Figure 1 . The adherence to the regular medicines was reported to be high or moderate by $70 \%$ $(n=74)$ of the participants, and the corresponding for oral spasmolytic drugs was $74 \%(n=29)$.

\section{Beliefs about medicines}

A total of $97 \%$ of the participants reported high perceptions of necessity to their treatment, while $54 \%$ reported a high level of concern. Most participants (65\%) believed that medicines are generally overused and $42 \%$ believed that medicines are generally harmful. Responses to the individual items on the BMQ-Concern and BMQ-Necessity scales are presented in Figure 2.

Categorizing the participants in four attitudinal groups based on data from the BMQ-Concern and BMQ-Necessity subscales showed that $52 \%$ of the participants were ambivalent (high necessity beliefs and high concerns) to their treatment while the other half was accepting, Figure 3.

\section{Variables associated with adherence}

Analysis of associations between adherence and gender, age, neurological level, AIS-score, time since injury, number of regularly used drugs, VAS, BMQ and mPSFS showed that the only statistically significant variable was age $(P=0,008)$. In the retrospective sensitivity 


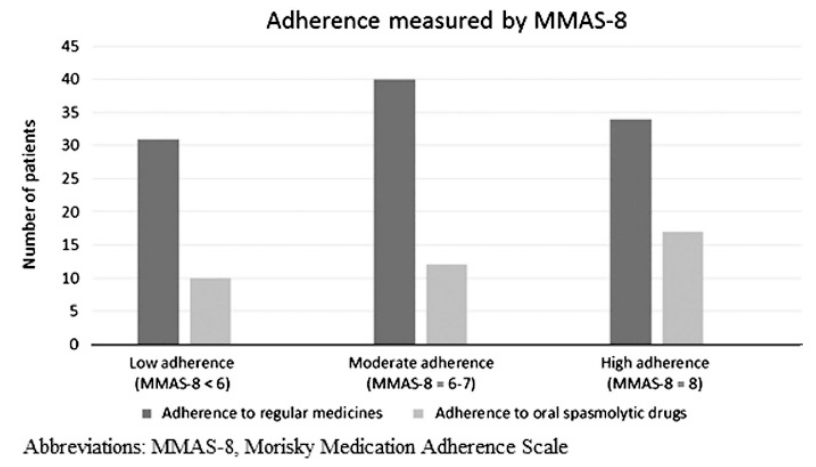

Figure $1 \mathrm{~A}$ histogram of adherence results from the MMAS-8. It shows percentages of patients being 'low adherent' (MMAS- $8=<6$ ), 'moderate adherent' (MMAS- $8=6-7$ ) or 'high adherent' (MMAS-8 $=8$ ) to both regular medicines and oral spasmolytics. $Y$ axis shows number of participants in each category.
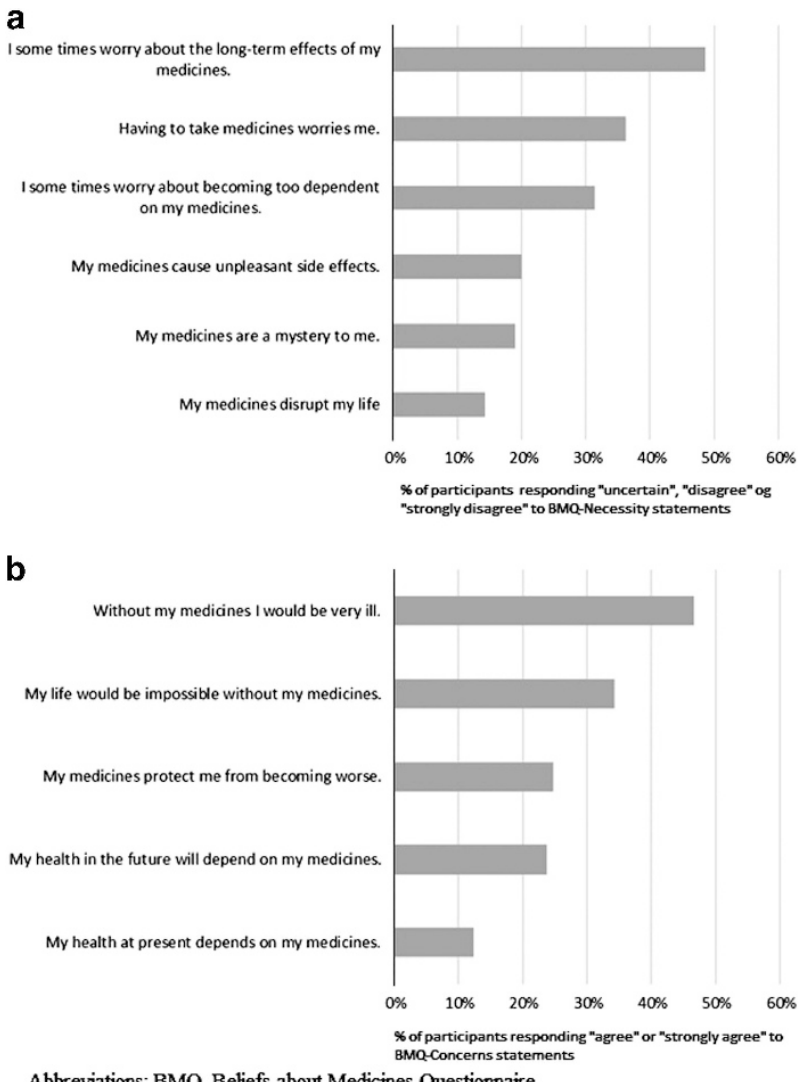

Figure 2 (a) Percentages of participants expressing concerns about their medicines, as indicated by responding 'uncertain', 'disagree' or 'strongly disagree' on BMQ-Necessity items. (b) Percentages of participants expressing doubts about their medicines, as indicated by responding 'agree' or 'strongly agree' to BMQ-Concerns item.

analysis on the other hand, using the classification by Biering-Sorensen et al., ${ }^{18}$ age was not formally a significant variable associated with adherence $(P=0,055)$.

\section{DISCUSSION}

To our knowledge, this is the first study describing the use of medicines and adherence among persons with chronic SCI. Our finding that the participants used in average 4.2 regular drugs is in line with other studies reporting on persons with chronic conditions using in average 3-5 regular drugs. ${ }^{19-21}$

Only a third of the participants in our cohort used oral spasmolytic drugs regularly, even though nearly $80 \%$ of them reported at least some level of spasticity. The explanation could be that mild spasticity can have a positive impact on functionality, or the fact that the most common oral spasmolytic drugs come with undesirable side effects as sedation and dizziness. ${ }^{7,22}$ Baclofen was the most frequently used API in our cohort, which in agreement with the fact that baclofen is the most common oral spasmolytic drug used by persons with chronic SCI. ${ }^{23}$

The finding that approximately two thirds of the patients used analgesics regularly underscore the pain challenge they have. This included pregabalin, gabapentin and amitriptyline, which we chose to register as analgesics, although they are not formally classified as belonging to this ATC-group. Pregabalin, gabapentin and amitriptyline were among the 10 most frequently used APIs in this population, and are known to be the most effective drugs in the treatment of neuropathic pain among persons with SCI. ${ }^{24}$ The majority of the drugs on demand were also analgesics, with paracetamol, tramadole and codeine being the most frequently used APIs. Despite the fact that about two thirds of the participants used analgesics regularly, almost everyone (94\%) reported that they experienced some level of pain at the time of the interview. The explanation could be that treatment of neuropathic pain is often difficult, and oral analgesics only give a small or moderate pain relief. ${ }^{24,25}$

The results show that over two thirds were highly or moderately adherent to their regular drugs, which is more than what has been found in previous studies with persons with other chronic conditions, where $11-56 \%$ of the persons had a high level of adherence. ${ }^{26-28}$ The adherence was also high for spasmolytic drugs. The high level of adherence could be explained by the seriousness of the condition or escalation of severe symptoms as pain or spasms when not following the medication regimen. The latter has been found to be associated with adherence in other studies. ${ }^{29}$ The only variable that was found to be associated with adherence was age, with younger age being associated with poorer adherence. This finding is supported by several studies with persons with other chronic conditions, suggesting that younger persons are more likely to be newly diagnosed and therefore less familiar with and in control of their diagnosis. ${ }^{30}$ It should be acknowledged that the small sample size in our study could explain the failure to find associations between the variables and adherence.

Although the association between age and adherence was significant, the finding was not formally confirmed by the retrospective sensitivity analysis using the age classification by Biering-Sorensen et al. ${ }^{18}$ This could be explained by the low number of participants in the youngest and oldest age groups in the latter analysis.

Compared to previous studies on long-term conditions, our persons with chronic SCI score higher when it comes to believing in the necessity of their treatment. On the other side, participants in our study were more concerned about their treatment and they also believed that medicines are more harmful and that there is more overuse than what has been found previously. ${ }^{31-34}$ Some studies suggest that persons who score high on the BMQ-Concern subscale are also likely to score higher on BMQ-Harm and BMQ-Overuse subscales. ${ }^{30}$ Our findings support this theory.

This study has some limitations in addition to those noted above. First, the majority of the results in this study are based on self-reported data, so we are not able to exclude reporting biases. The interview guide used in this study was not validated, only tested in five participants in a pilot study. Although MMAS-8 and BMQ are 


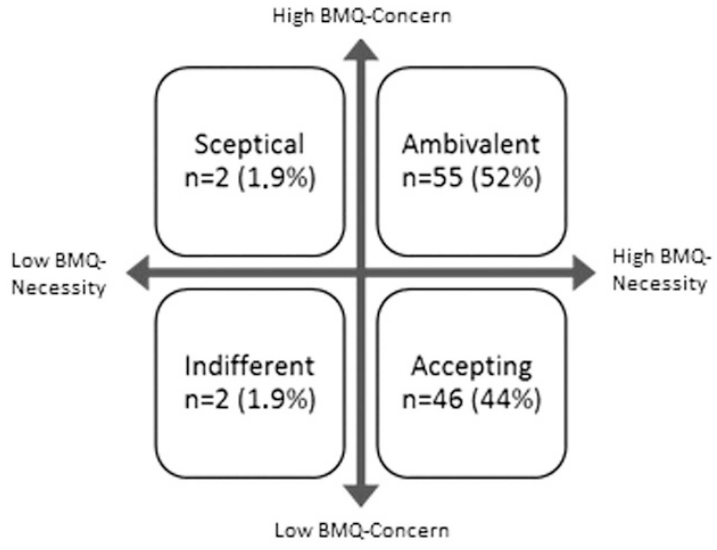

Abbreviations: BMQ, Beliefs about Medicines Questionnaire

Figure 3 Patients divided in attitudinal groups 'accepting' (high necessity and low concern), 'ambivalent' (high necessity and high concern), 'indifferent' (low necessity and low concern) and 'sceptical' (low necessity and high concern) based on BMQ-Concern and BMQ-Necessity results.

validated for several other patient groups, they have not been validated for persons with SCI. It should be mentioned that self-report questionnaires have in previous studies been associated with overreporting of adherence. ${ }^{35}$ Further, the participants who needed assistance for completing the questionnaires may not have answered as honestly as those who completed the questionnaires by themselves.

The participants were not asked to report the indication for the drugs they were using. Therefore, it is not certain if all the medicines listed are used as a result of a SCI or because of other conditions. And lastly, it is possible the group of persons studied may not be generalizable to the whole population of persons with chronic SCI. We only included persons admitted for a voluntary follow-up stay, who might have a better insight into their condition and are more in control of their treatment compared to the rest of this population. On the other hand, some eligible persons might be too busy to participate as well. This could contribute to explaining the high levels of adherence and beliefs of necessity reported by the participants in the present study. Thus, additional studies are required and should strive to include a broader patient population, also including post-acute injured persons.

\section{CONCLUSION}

This study indicates that persons with chronic SCI use as many regular drugs as persons with other chronic conditions. Most of the participants in our group find their medicines necessary, although the majority also had high levels of concern to their medicines. This indicates that there is need for more education and counseling, for example, in forms of medicine use reviews, to make the participants feel safe about their drug regimen. Our findings also suggest that our cohort have higher overall adherence compared to other patient groups with chronic illnesses. There is need for further studies with larger population groups to understand adherence and factors determining adherence among persons with SCI.

\section{DATA ARCHIVING}

There were no data to deposit.

\section{CONFLICT OF INTEREST}

The authors declare no conflict of interest.

\section{ACKNOWLEDGEMENTS}

The study was funded by the Hospital Pharmacies Enterprise, South Eastern Norway and Sunnaas Rehabilitation Hospital. We thank the study participants and the nursing staff of department for follow-up at Sunnaas Rehabilitation Hospital.

1 Garstang SV, Miller-Smith SA. Autonomic nervous system dysfunction after spinal cord injury. Phys Med Rehab Clin North Am 2007; 18: 275-296.

2 Hsieh JT, Wolfe DL, Miller WC, Curt A. Spasticity outcome measures in spinal cord injury: psychometric properties and clinical utility. Spinal Cord 2008; 46: 86-95.

3 Hagen EM, Rekand T. Management of neuropathic pain associated with spinal cord injury. Pain Ther 2015; 4: 51-65.

4 World Health Organization. Adherence to long-term therapies-Evidence for action. Geneva, Switzerland, 2003. Available at http://www.who.int/chp/knowledge/publications/adherence_full_report.pdf (accessed 23 June 2017).

5 Nguyen TM, La Caze A, Cottrell N. What are validated self-report adherence scales really measuring?: a systematic review. Br J Clin Pharmacol 2014; 77: 427-445.

6 Adams MM, Hicks AL. Spasticity after spinal cord injury. Spinal Cord 2005; 43 : 577-586.

7 Halpern R, Gillard P, Graham GD, Varon SF, Zorowitz RD. Adherence associated with oral medications in the treatment of spasticity. J Injury Funct Rehab 2013; 5: 747-756.

8 Sunnaas sykehus HF, Haukeland Universitetssykehus HF, St. Olavs Hospital HF. ABC om ryggmargsskade for helsepersonell. Landsforeningen for Ryggmargsskadde. Oslo, 2012. Available at http://lars.no/vare-publikasjoner (accessed 23 June 2017).

9 Scullin C, Scott MG, Hogg A, McElnay JC. An innovative approach to integrated medicines management. J Eval Clin Pract 2007; 13: 781-788.

10 WHO Collaborating Centre for Drug Statistic Methodology. Anatomical Therapeutic Chemical Code. WHO: Oslo, Norway. 2013. Available at http://www.whocc.no (accesses 23 June 2017).

11 Morisky DE, DiMatteo MR. Improving the measurement of self-reported medication nonadherence: final response. J Clin Epidemiol 2011; 64: 262-263.

12 Morisky DE, Ang A, Krousel-Wood M, Ward HJ. Predictive validity of a medication adherence measure in an outpatient setting. J Clin Hypertens 2008; 10: 348-354.

13 Krousel-Wood M, Islam T, Webber LS, Re RN, Morisky DE, Muntner P. New medication adherence scale versus pharmacy fill rates in seniors with hypertension. Am J Manag Care 2009; 15: 59-66.

14 Horne R, Weinman J, Hankins M. The beliefs about medicines questionnaire: the development and evaluation of a new method for assessing the cognitive representation of medication. Psychol Health 1999; 14: 1-24.

15 McCormack HM, de L Horne DJ, Sheather S. Clinical applications of visual analogue scales: a critical review. Psychol Med 2009; 18: 1007-1019.

16 Collins SL, Moore RA, McQuay HJ. The visual analogue pain intensity scale: what is moderate pain in millimetres? Pain 1997; 72: 95-97.

17 Penn RD. Intrathecal Baclofen for severe spasticity. Ann N Y Acad Sci 1988; 531: 157-166.

18 Biering-Sørensen F, DeVivo MJ, Charlifue S, Chen Y, New PW, Noonan V et al. International spinal cord injury core data set (version 2.0)-including standardization of reporting. Spinal Cord 2017; 55: 759-764.

19 Napolitano F, Napolitano P, Angelillo IF. Medication adherence among patients with chronic conditions in Italy. Eur J Public Health 2016; 26: 48-52.

20 Akincigil A, Bowblis JR, Levin C, Walkup JT, Jan S, Crystal S. Adherence to antidepressant treatment among privately insured patients diagnosed with depression. Med Care 2007: 45: 363-369.

21 Nilsson N, Lea M, Lao Y, Wendelbo K, Gløersen G, Mowé M et al. Medication discrepancies revealed by medication reconciliation and their potential short-term and long-term effects: a Norwegian multicentre study carried out on internal medicine wards. Eur J Hosp Pharm 2015; 22: 298-303.

22 Stevenson VL. Rehabilitation in practice: spasticity management. Clin Rehab 2010; 24: 293-304.

23 Rekand T, Hagen EM, Gronning M. Spasticity following spinal cord injury. Tidsskrift for den Norske laegeforening 2012; 132: 970-973.

24 Finnerup NB, Baastrup C, Jensen TS. Neuropathic pain following spinal cord injury pain: mechanisms and treatment. Scand J Pain 2009; 1: S3-S11.

25 Baastrup C, Finnerup NB. Pharmacological management of neuropathic pain following spinal cord injury. CNS Drugs 2008; 22: 455-475.

26 Saadat Z, Nikdoust F, Aerab-Sheibani H, Bahremand M, Shobeiri E, Saadat H et al. Adherence to antihypertensives in patients with comorbid condition. Nephro Urol Month 2015; 7: e29863.

27 Castellucci LA, Shaw J, van der Salm K, Erkens P, Le Gal G, Petrcich W et al. Selfreported adherence to anticoagulation and its determinants using the Morisky medication adherence scale. Thromb Res 2015; 136: 727-731.

28 Morisky DE, Malotte CK, Choi P, Davidson P, Rigler S, Sugland B et al. A patient education program to improve adherence rates with antituberculosis drug regimens. Health Educ Q 1990; 17: 253-267.

29 Osterberg L, Blaschke T. Adherence to medication. N Engl J Med 2005; 353: 487-497. 
30 Krueger K, Botermann L, Schorr SG, Griese-Mammen N, Laufs U, Schulz M. Age related medication adherence in patients with chronic heart failure: a systematic literature review. Int J Cardiol 2015; 184: 728-735.

31 Horne R, Weinman J. Patients' beliefs about prescribed medicines and their role in adherence to treatment in chronic physical illness. J Psychosom Res 1999; 47: 555-567.

32 Maguire LK, Hughes CM, McElnay JC. Exploring the impact of depressive symptoms and medication beliefs on medication adherence in hypertension-a primary care study. Patient Educ Counsel 2008; 73: 371-376.
33 Viktil KK, Froyland H, Rogvin M, Moger TA. Beliefs about medicines among Norwegian outpatients with chronic cardiovascular disease. Eur J Hosp Pharm 2014; 21: $118-120$.

34 Neame R, Hammond A. Beliefs about medications: a questionnaire survey of people with rheumatoid arthritis. Rheumatology 2005; 44: 762-767.

35 Stirratt MJ, Dunbar-Jacob J, Crane HM, Simoni JM, Czajkowski S, Hilliard ME et al. Self-report measures of medication adherence behavior: recommendations on optimal use. Trans/ Behav Med 2015; 5: 470-482. 